\title{
Three-Dimensional General-Relativistic Magnetohydrodynamic Simulations of Remnant Accretion Disks from Neutron Star Mergers: Outflows and $r$-Process Nucleosynthesis
}

\author{
Daniel M. Siegel ${ }^{*}$ and Brian D. Metzger \\ Physics Department and Columbia Astrophysics Laboratory, Columbia University, New York, New York 10027, USA
}

(Received 16 May 2017; revised manuscript received 22 September 2017; published 6 December 2017)

\begin{abstract}
The merger of binary neutron stars, or of a neutron star and a stellar-mass black hole, can result in the formation of a massive rotating torus around a spinning black hole. In addition to providing collimating media for $\gamma$-ray burst jets, unbound outflows from these disks are an important source of mass ejection and rapid neutron capture ( $r$-process) nucleosynthesis. We present the first three-dimensional generalrelativistic magnetohydrodynamic (GRMHD) simulations of neutrino-cooled accretion disks in neutron star mergers, including a realistic equation of state valid at low densities and temperatures, self-consistent evolution of the electron fraction, and neutrino cooling through an approximate leakage scheme. After initial magnetic field amplification by magnetic winding, we witness the vigorous onset of turbulence driven by the magnetorotational instability (MRI). The disk quickly reaches a balance between heating from MRI-driven turbulence and neutrino cooling, which regulates the midplane electron fraction to a low equilibrium value $Y_{e} \approx 0.1$. Over the 380 -ms duration of the simulation, we find that a fraction $\approx 20 \%$ of the initial torus mass is unbound in powerful outflows with asymptotic velocities $v \approx 0.1 c$ and electron fractions $Y_{e} \approx 0.1-0.25$. Postprocessing the outflows through a nuclear reaction network shows the production of a robust second- and third-peak $r$ process. Though broadly consistent with the results of previous axisymmetric hydrodynamical simulations, extrapolation of our results to late times suggests that the total ejecta mass from GRMHD disks is significantly higher. Our results provide strong evidence that postmerger disk outflows are an important site for the $r$ process.
\end{abstract}

DOI: $10.1103 /$ PhysRevLett.119.231102

Introduction.-Approximately half of the elements heavier than iron are synthesized by the capture of neutrons onto lighter seed nuclei in a dense neutron-rich environment in which the time scale for neutron capture is shorter than the $\beta$-decay time scale $[1,2]$. This "rapid neutroncapture process" ( $r$ process) occurs along a nuclear path far on the neutron-rich side of the valley of stable isotopes. Despite this realization 70 years ago, the identity of the astrophysical sites giving rise to the $r$ process remains an enduring mystery [3-5].

Among the promising $r$-process sites are the mergers of compact binaries consisting of two neutron stars (NS-NS, BNS) [6] or of a NS and stellar-mass black hole (NS-BH) [7]. These violent events produce several sources of neutron-rich ejecta, which contribute to their total $r$-process yields $[8,9]$. Historically, most work has focused on matter ejected during the merger process itself, either by tidal forces or due to shock and compression-induced heating at the interface between merging bodies [10-16]. While there is broad agreement that a portion of this "dynamical ejecta" is sufficiently neutron-rich to create the heaviest $r$-process elements, its quantity is sensitive to the properties of the merging binary and the NS equation of state (EOS).

NS mergers are also accompanied by the formation of a massive accretion disk surrounding the central compact object (e.g., [17,18]). Soon after forming, the neutrino luminosity of the disk is high [19], driving a small quantity of mass from the disk surface in a neutrino-driven wind [20-25].

On longer time scales of hundreds of milliseconds, the disk expands radially due to the outwards transport of angular momentum. One-dimensional models of this spreading evolution using an $\alpha$ prescription for the effective turbulent viscosity $[21,26]$ showed that, as the disk accretion rate drops, the midplane transitions from a neutrinocooled state to a radiatively inefficient one [27,28]. Powerful outflows were predicted following this transition, once heating from turbulent dissipation and nuclear recombination (chiefly $\alpha$-particle formation) are no longer balanced by neutrino cooling.

These initial models were followed by two-dimensional hydrodynamical simulations of the disk evolution in a pseudo-Newtonian gravitational potential, which also adopted an $\alpha$-viscosity prescription. References [29] and [30] employed an approximate leakage scheme to account for neutrino cooling and a "light-bulb" irradiation model for the neutrino heating, while [31] used an energy-dependent twomoment closure scheme for the transport of electron neutrinos and antineutrinos. These works found unbound outflows with electron fractions in the range $Y_{e} \sim 0.2-0.4$ [29,31], sufficient to produce the entire mass range of $r$-process elements [31-33]. The total fraction of the original disk mass unbound in outflows ranged from $\sim 5 \%$ for a nonspinning $\mathrm{BH}$, to $\sim 30 \%$ for high $\mathrm{BH}$ spin $\chi_{\mathrm{BH}} \simeq 0.95[30,31]$. 
Previous simulations of the remnant disk employ a parameterized hydrodynamical viscosity in place of a self-consistent physical mechanism for angular momentum transport as mediated by the magnetorotational instability (MRI) [34]. Reference [35] performed two-dimensional general-relativistic magnetohydrodynamic (GRMHD) simulations of the disk evolution lasting $60 \mathrm{~ms}$; however, they were not focused on nucleosynthesis and their restriction to 2D precluded a study of the saturated MRI due to the antidynamo theorem. In this Letter, we present the first fully three-dimensional GRMHD simulations of the remnant accretion disk evolution and its outflows over a time scale of $\approx 400$ ms following the merger.

Numerical setup and initial conditions.-Simulations are performed in ideal GRMHD with a fixed background spacetime using the open-source EINSTEINTOOLKIT [36,37] with the GRMHD code GRHYDRO [38]. GRMHD is implemented using a finite-volume scheme with piecewise parabolic reconstruction [39], the Harten-Lax-van Leer-Einfeldt Riemann solver [40,41], and constrained transport [42] for maintaining the magnetic field divergence free. We have implemented a new framework for the recovery of primitive variables in GRHYDRO that provides support for any threeparameter EOS, as well as a recovery scheme based on threedimensional root finding according to [43], which shows better and faster convergence properties than two-dimensional schemes; its ability to recover strongly magnetized regions is important for evolving low-density, magnetized disk winds.

Thermodynamic properties of matter are described by the Helmholtz EOS $[44,45]$, which includes contributions to the Helmholtz free energy from nuclei (treated as ideal gas) with Coulomb corrections, electrons and positrons with an arbitrary degree of relativity and degeneracy, and photons in local thermodynamic equilibrium. We consider free neutrons, protons, and $\alpha$ particles, whose abundances are calculated assuming nuclear statistical equilibrium (NSE). We add dissociation energies to the Helmholtz EOS as in [29] to account for the energy release from $\alpha$-particle formation, as well as the additional terms to the thermodynamic derivatives arising from compositional changes.

Neutrino cooling is described by a leakage scheme newly implemented into GRHYDRO. Leakage schemes are widely used in both core-collapse supernovae and compact-binary merger simulations (e.g., [46-51]). Our implementation follows closely Ref. [16], which is based on [52] and employs the formalism by [47]. We calculate optical depths following the procedure by [53], which is well suited for the aspherical geometry of an accretion disk. We neglect neutrino absorption, which is expected to appreciably change the outflow dynamics only for significantly more massive accretion disks [29] (see also Fig. 1).

Initial data consist of an equilibrium torus of constant specific angular momentum and specific entropy around a Kerr BH $[54,55]$ (Table I). We compute a torus solution in

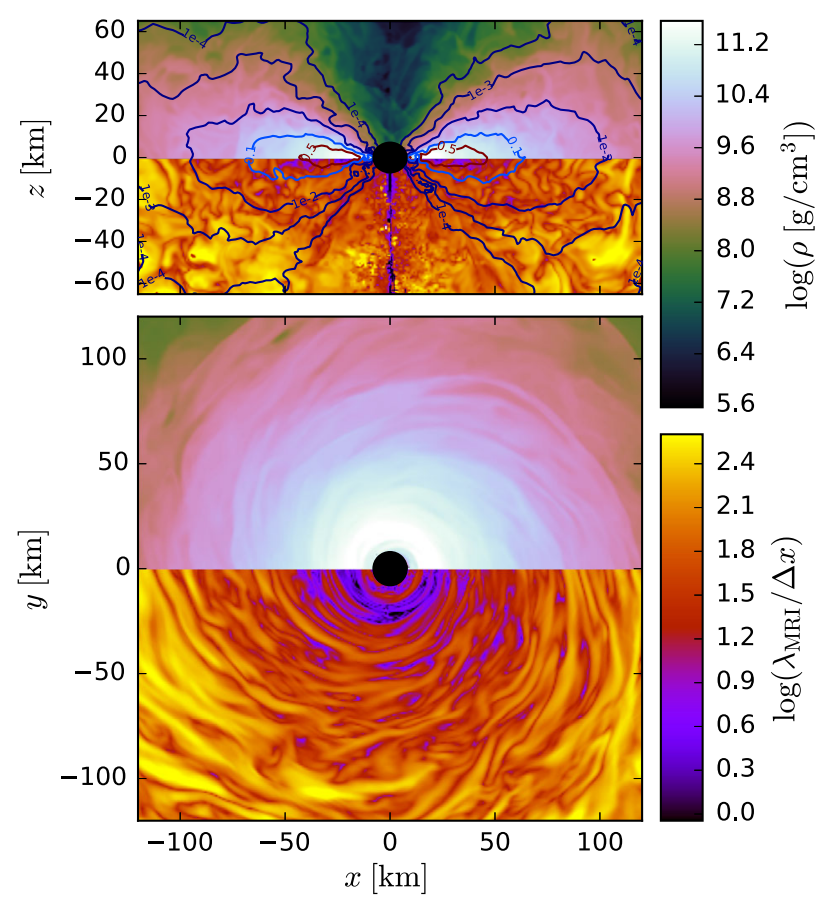

FIG. 1. Snapshots of rest-mass density, number of grid points per fastest-growing MRI mode, and contours of optical depth to electron neutrino number emission $\tau_{\nu_{e}}=0.5,0.1,10^{-2}, 10^{-3}$, $10^{-4}$ at $t=20 \mathrm{~ms}$, when the disk has settled into a quasistationary state (the $\mathrm{BH}$ interior is masked).

horizon-penetrating Kerr-Schild coordinates, which we use in our simulation. The $\mathrm{BH}$ mass and spin correspond to a typical NS merger remnant. BHs promptly formed in BNS mergers show spins $\chi_{\mathrm{BH}} \approx 0.8$ [56-58], and are unlikely to be significantly larger [59], whereas BHs formed by delayed collapse typically show spins $\chi_{\mathrm{BH}} \lesssim 0.7$ [60]. Furthermore, $\chi_{\mathrm{BH}} \sim 0.8$ also represents a typical $\mathrm{BH}$ spin for BH-NS mergers required to tidally disrupt the NS and form a sufficiently massive torus [61]. The initial torus mass also corresponds to typical NS merger scenarios (e.g., [62,63]). We set up a weak initial magnetic seed field inside the torus with vector potential components $A^{r}=A^{\theta}=0$ and $A^{\phi}=A_{b} \max \left\{p-p_{\text {cut }}, 0\right\}$. Here, $p$ denotes the fluid pressure, $p_{\text {cut }}=1.3 \times 10^{-2} p_{\max }$, where $p_{\max }$ is the pressure at maximum density in the torus, and $A_{b}$ sets the initial field strength, which we adjust such that the maximum magneticto-fluid pressure ratio in the torus is $<5 \times 10^{-3}$; this results in a maximum magnetic field strength of $\approx 3.3 \times 10^{14} \mathrm{G}$. The

TABLE I. Initial configuration: $\mathrm{BH}$ mass and dimensionless spin, torus mass, inner and outer torus radius, radius at maximum density, specific entropy, electron fraction, and maximum magnetic-to-fluid pressure ratio.

\begin{tabular}{lcccccccc}
\hline \hline $\begin{array}{l}M_{\mathrm{BH}} \\
\left(M_{\odot}\right)\end{array}$ & $\chi_{\mathrm{BH}}$ & $\begin{array}{c}M_{t 0} \\
\left(M_{\odot}\right)\end{array}$ & $\begin{array}{c}R_{\mathrm{in}, 0} \\
\left(M_{\mathrm{BH}}\right)\end{array}$ & $\begin{array}{c}R_{\mathrm{out}, 0} \\
\left(M_{\mathrm{BH}}\right)\end{array}$ & $\begin{array}{c}R_{0} \\
(\mathrm{~km})\end{array}$ & $\begin{array}{c}s_{0} \\
\left(k_{B} / \mathrm{b}\right)\end{array}$ & $Y_{e 0}$ & $p_{b} / p_{f}$ \\
\hline 3.00 & 0.8 & 0.03 & 4 & 24 & 30 & 8 & 0.1 & $<5 \times 10^{-3}$ \\
\hline \hline
\end{tabular}


TABLE II. Initial torus mass after early relaxation and characteristics of the disk outflows as measured by tracer particles: mean electron fraction, specific entropy, expansion time scales, all at $t=t_{5} \mathrm{GK}$ and subdivided into equatorial $\left(30^{\circ}<\theta<150^{\circ}\right)$ and polar $\left(\theta \leq 30^{\circ}\right.$ and $\theta \geq 150^{\circ}$ ) outflow (the polar angle $\theta$ being measured at the end of the simulation), as well as total integrated outflow mass (polar or equatorial and total). Corresponding values by [30] (F15) and [31] (J15) are also listed.

\begin{tabular}{|c|c|c|c|c|c|c|c|c|c|c|c|c|c|}
\hline \multirow[b]{2}{*}{ Simulation } & \multirow[b]{2}{*}{ Outflow type } & \multirow[b]{2}{*}{$\begin{array}{c}M_{t, \text { in }} \\
\left(10^{-2} M_{\odot}\right)\end{array}$} & \multicolumn{3}{|c|}{ Equatorial outflow } & \multicolumn{3}{|c|}{ Polar outflow } & \multicolumn{3}{|c|}{ Total outflow } & \multirow[b]{2}{*}{$\begin{array}{l}M_{\mathrm{pol}} \\
\left(M_{\mathrm{eq}}\right)\end{array}$} & \multirow[b]{2}{*}{$\begin{array}{c}M_{\text {out }} \\
\left(M_{t, \text { in }}\right)\end{array}$} \\
\hline & & & $\bar{Y}_{e}$ & $\begin{array}{c}\bar{s} \\
\left(k_{B} / \mathrm{b}\right)\end{array}$ & $\begin{array}{l}\bar{t}_{\text {exp }} \\
(\mathrm{ms})\end{array}$ & $\bar{Y}_{e}$ & $\begin{array}{c}\bar{s} \\
\left(k_{B} / \mathrm{b}\right) \\
\end{array}$ & $\begin{array}{l}\bar{t}_{\text {exp }} \\
(\mathrm{ms})\end{array}$ & $\bar{Y}_{e}$ & $\begin{array}{c}\bar{s} \\
\left(k_{B} / \mathrm{b}\right)\end{array}$ & $\begin{array}{l}\bar{t}_{\mathrm{exp}} \\
(\mathrm{ms})\end{array}$ & & \\
\hline This work & Unbound & 2.02 & 0.18 & 31 & 24 & 0.19 & 39 & 18 & 0.18 & 32 & 23 & 0.22 & 0.16 \\
\hline This work & Total & 2.02 & 0.17 & 28 & 26 & 0.19 & 43 & 18 & 0.17 & 30 & 25 & 0.15 & 0.23 \\
\hline F15 t-a80 & Total & 3.00 & 0.22 & 21 & 35 & 0.31 & 38 & 9.4 & $\ldots$ & $\ldots$ & $\ldots$ & 0.01 & 0.17 \\
\hline $\mathrm{J} 15 \mathrm{M} 3 \mathrm{~A} 8 \mathrm{~m} 03 \mathrm{a} 2$ & Total & 3.00 & $\ldots$ & $\ldots$ & $\ldots$ & $\ldots$ & $\ldots$ & $\ldots$ & 0.27 & 30 & $\ldots$ & $\ldots$ & 0.23 \\
\hline J15 M3A8m03a5 & Total & 3.00 & $\cdots$ & $\ldots$ & $\cdots$ & $\cdots$ & $\ldots$ & $\ldots$ & 0.25 & 33 & $\ldots$ & $\cdots$ & 0.24 \\
\hline
\end{tabular}

torus is initially embedded in a uniform, tenuous atmosphere with $\rho \approx 37 \mathrm{~g} \mathrm{~cm}^{-3}, T=10^{5} \mathrm{~K}$, and $Y_{e}=1$. This density and temperature are sufficiently low to impact neither the dynamics nor the composition of the disk outflows.

Simulations are performed in full 3D without employing symmetries. The grid setup consists of a Cartesian grid hierarchy of eight refinement levels, extending from the center of the $\mathrm{BH}$ to $1.53 \times 10^{9} \mathrm{~cm}$ in every coordinate direction. The finest refinement level corresponds to a spatial domain with a resolution of $856 \mathrm{~m}$ and a diameter of $240 \mathrm{~km}$, which entirely contains the initial accretion torus.

Results.-The initial torus is evolved from $t=0$ to $t=381 \mathrm{~ms}$, after which an appreciable fraction of the initial torus mass has been unbound in powerful outflows. After an initial transient phase of about $20 \mathrm{~ms}$ due to the onset of turbulence created by the MRI, the disk settles into a quasistationary state for the rest of the simulation. During this early relaxation, $\approx 33 \%$ of the initial torus mass is either accreted onto the $\mathrm{BH}$ or ejected via outflows, leaving an effective initial torus of $\approx 0.02 M_{\odot}$ (Table II). We exclude matter ejected or accreted during this phase from all further analysis.

Figure 1 shows snapshots of several quantities at the beginning of the quasistationary evolution phase. Until the end of the simulation, the disk and outflows remain qualitatively similar as depicted here. In particular, the disk remains optically thin with respect to neutrinos, which have typical energies of a few MeV (Fig. 1, left, upper panel). Neutrino cooling mainly acts in regions closely to the disk midplane, as neutrino emission tracks density. Matter in the disk is heated as it gradually falls into the $\mathrm{BH}$ potential due to angular momentum transport via MHD turbulence mediated by the MRI. We check that the MRI is well resolved by monitoring the wavelength of the fastest-growing MRI mode, $\lambda_{\text {MRI }}$, which is typically resolved by 10 or more grid points (cf. Fig. 1, left); $\lambda_{\mathrm{MRI}}$ is estimated by $\lambda_{\mathrm{MRI}}=$ $(2 \pi / \Omega)\left(b / \sqrt{4 \pi \rho h+b^{2}}\right)$ [64], where $\Omega$ is the angular frequency, $\rho$ the rest-mass density, $h$ the specific enthalpy, and $b=\sqrt{b^{\mu} b_{\mu}}$ the comoving magnetic field strength. Very close to the $\mathrm{BH}$ resolving the MRI becomes challenging with current computational resources, and $\lambda_{\mathrm{MRI}}$ is not resolved by $>10$ grid points at all times and spatial points. At the beginning of the simulation, after initial amplification by magnetic winding, the onset of the MRI further amplifies the weak initial seed magnetic field in the disk over a few rotational periods (resulting in a total amplification of roughly 2 orders of magnitude for the maximum field strength), before the disk settles into a saturated MRI state. Triggering the MRI both in the poloidal and toroidal components entirely without magnetic winding (for the same initial seed field strength) would require higher resolution and would thus be challenging with current computational resources; this simulation only represents a first attempt in this direction. We note that the resulting typical magnetic field strengths of up to $\sim 10^{15} \mathrm{G}$ close to the $\mathrm{BH}$ and the midplane, and typical magnetic-to-fluid pressure ratios of $\sim 10^{-3}-10^{-1}$, are similar to values found in early BNS postmerger accretion systems [64,65]. Figure 2 shows the evolution of the (density-averaged) ratio of electromagnetic energy to internal energy of the disk, $\left\langle e_{\mathrm{EM}} / e_{\text {int }}\right\rangle_{D}=$ $\left\langle n_{\mu} n_{\nu} T_{\mathrm{EM}}^{\mu \nu} / \epsilon \rho W\right\rangle_{D}$, where $n^{\mu}$ denotes the unit normal to the spatial hypersurfaces of the spacetime foliation, $T_{\mathrm{EM}}^{\mu \nu}$ the stress-energy tensor of the electromagnetic field, $\epsilon$ the specific internal energy, $W$ the Lorentz factor, and

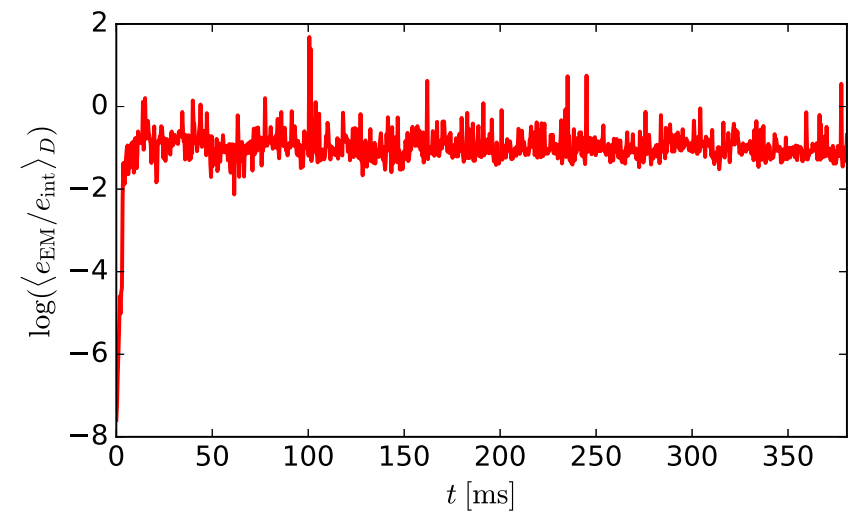

FIG. 2. Evolution of the density-averaged ratio of electromagnetic energy to internal energy in the disk. 


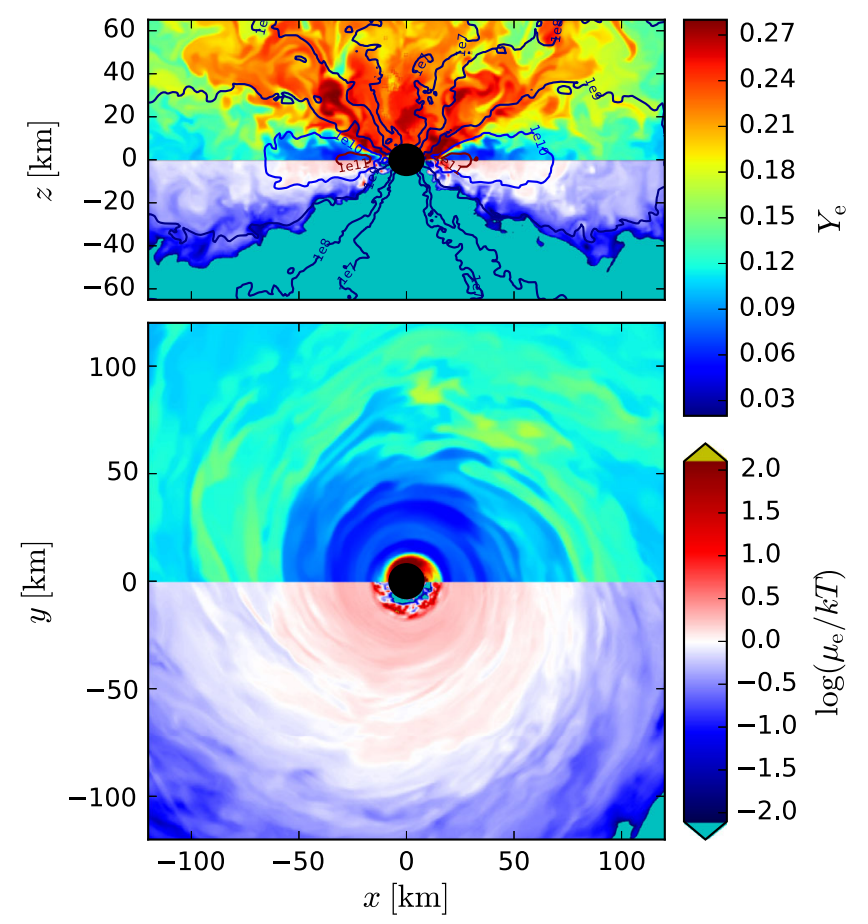

FIG. 3. Snapshots of electron fraction, normalized electron chemical potential, and contours of rest-mass density $\rho=$ $\left[10^{7}, 10^{8}, 10^{9}, 10^{10}, 10^{11}\right] \mathrm{g} \mathrm{cm}^{-3}$ at $t=43 \mathrm{~ms}$, when the disk has fully self-regulated itself to mild electron degeneracy (the $\mathrm{BH}$ interior is masked).

$\langle\cdots\rangle_{D} \equiv \int \cdots D d^{3} x / \int D d^{3} x$, with $D=\sqrt{\gamma} \rho W$ the conserved rest-mass density and $\gamma$ the determinant of the spatial metric. This ratio stays roughly constant for $t>20 \mathrm{~ms}$ (in a time-averaged sense) and thus indicates that a steady turbulent state of the disk is indeed achieved.

Optically thin neutrino cooling in the midplane is balanced by MHD-driven heating, and the disk regulates itself to a mildly degenerate state with low $Y_{e}$ [66]. The latter results from a negative feedback process: higher electron degeneracy $\mu_{e} / k_{B} T$ results in less electrons (lower $Y_{e}$ ) and positrons, causing less neutrino emission, i.e., a lower cooling rate, therefore higher temperatures, and thus lower degeneracy; the resulting state is independent of the initial conditions. Figure 3 shows the disk once it has fully self-regulated itself into this mildly degenerate state $\left(\mu_{e} / k_{B} T \sim 1\right)$. The inner disk remains neutron-rich $\left(Y_{e} \approx 0.1\right.$ ) over the course of the simulation up to radii $r \lesssim 60 \mathrm{~km} \quad(\lesssim 14$ gravitational radii), consistent with previous one-dimensional models of neutrino-cooled disks [26,67].

Above the disk midplane powerful thermal outflows are generated. These are the result of a heating-cooling imbalance: in regions of lower density, viscous heating from MHD-driven turbulence and energy release from recombination of free nucleons into $\alpha$ particles exceeds cooling by neutrino emission, and the weak interactions essentially "freeze out" (although further mixing can still change $\left.Y_{e}\right)$. In the polar funnel these outflows possess high$Y_{e}(>0.2)$ and high specific entropy $\left(s \gtrsim 100 k_{B} / \mathrm{b}\right)$, while the denser equatorially directed outflows have lower specific entropy $\left(\sim 10 k_{B} / \mathrm{b}\right)$ and lower $Y_{e}$.

Thermodynamic properties of the outflow are recorded by $10^{4}$ passive tracer particles that are advected with the fluid. We place these tracer particles of equal mass in the initial setup with a probability proportional to the conserved rest-mass density $D=\sqrt{\gamma} \rho W$. Table II and Fig. 4 characterize the outflow properties relevant to the $r$ process, including $Y_{e}, s$, and the expansion time scale $t_{\exp }=r / v$, where $v$ denotes the three-velocity (e.g., [68]). These quantities are evaluated for each tracer particle at the last time $t=t_{5 \mathrm{GK}}$ when the temperature of the particle drops below 5 GK. At 5 GK, NSE breaks down and full nuclear reaction network calculations are required to track nuclear abundances. We distinguish between total outflow, defined as all tracer particles that have reached $r \geq 10^{3} \mathrm{~km}$ by the end of the simulation, and unbound outflow, defined as those that are additionally unbound according to the Bernoulli criterion $-h u_{t}>1$, where $u_{t}$ is the time component of the four-velocity.

By the end of the simulation, $\approx(16-23) \%$ of the initial disk mass has been ejected into unbound outflows with $v \approx(0.03-0.1) c$ and asymptotic speeds of $v \approx 0.1 c$ after conversion of residual specific enthalpy to kinetic energy. With the disk still launching outflows by the end of the simulation, our GRMHD setup potentially unbinds significantly more mass compared to two-dimensional,
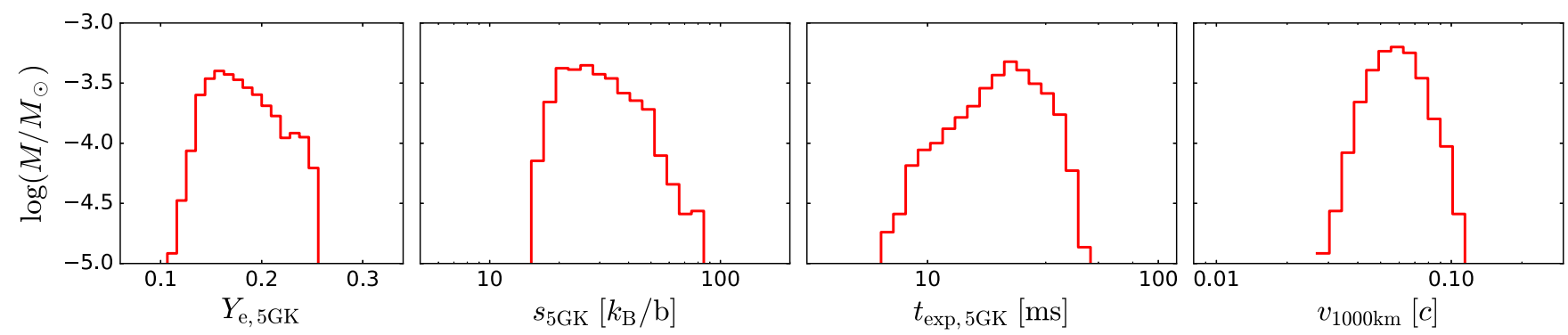

FIG. 4. Mass distributions of the unbound disk outflow as measured by tracer particles in terms of electron fraction, specific entropy, expansion time scale (all at $t=t_{5 \mathrm{GK}}$ ), and outflow velocity at $r=10^{3} \mathrm{~km}$. 
non-MHD, Newtonian simulations with similar disk parameters (Table II) [30,31]. Polar outflows show higher $Y_{e}$ and specific entropy, and smaller $t_{\exp }$ than equatorial outflows, consistent with [30], while we find a factor $\sim 20$ higher mass in polar outflows. Our $Y_{e}$ distribution shows a smaller mean and does not extend as high as in [30,31]. This may indicate that neutrino absorption (not included here) plays a dominant role in setting the high- $Y_{\mathrm{e}}$ tail of the distribution. Indeed, a preliminary reanalysis including effects of neutrino absorption as in [69] shows the ejecta achieves a high- $Y_{e}$ tail extending up to $\gtrsim 0.3$; however, our finding of a sizable quantity of low- $Y_{e}$ ejecta, capable of a full (second- and third-peak) $r$ process, remains robust. Alternatively, previously employed pseudo-Newtonian potentials and $\alpha$ disks may not accurately capture the heating-cooling interplay which also controls the evolution of $Y_{e}$.

Full nuclear reaction network calculations with SKYNET [68] were performed on the tracer particles in a postprocessing step, starting at $t=t_{10 \mathrm{GK}}$. Figure 5 shows that the solar abundances [70] are well reproduced throughout the mass number $(A)$ range from the second $r$-process peak
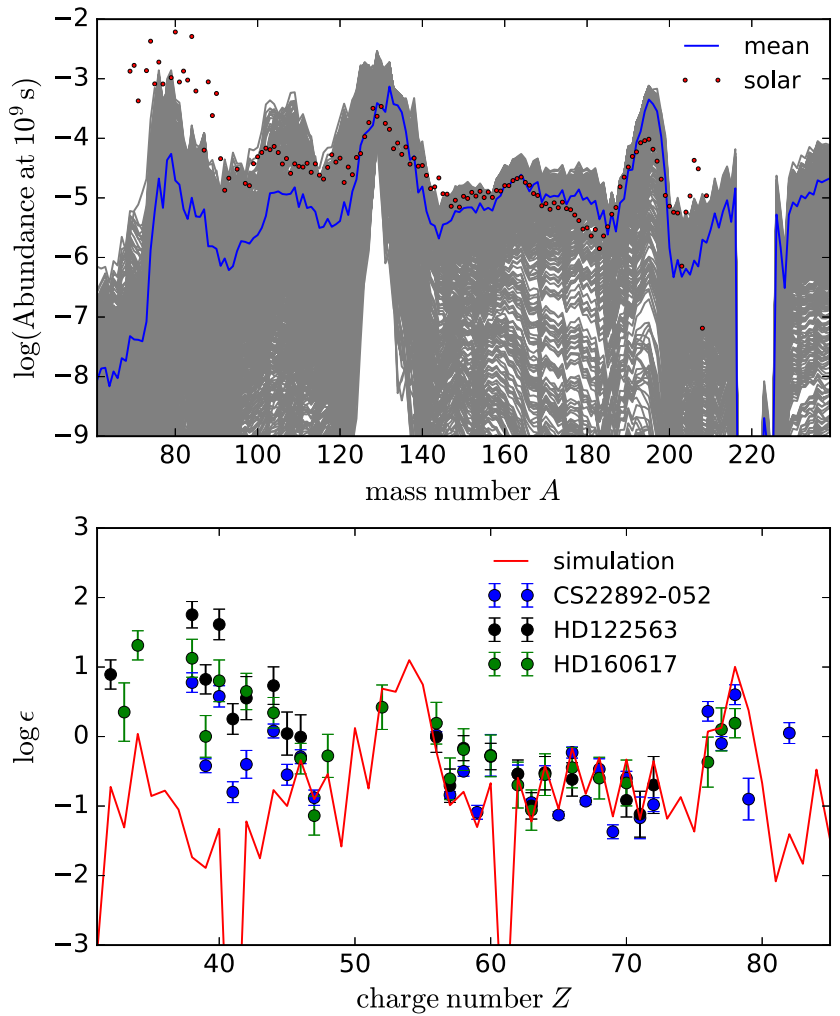

FIG. 5. Top: Final elemental abundances from individual unbound tracer particles (gray lines) and their mean (blue line), compared to observed solar system abundances [70], scaled to match the mean at $A=130$. Bottom: Comparison of the mean abundances to observed abundances in metal-poor halo stars [71-73] in terms of $\log \epsilon=\log Y_{Z} / Y_{1}+12$, scaled such that $\sum\left(\log Y_{Z} / Y_{Z, \mathrm{CS} 22892-052}\right)^{2}$ is minimized between $55 \leq Z \leq 75$.
$(A \sim 130)$ to the rare-earth peak $(A \sim 165)$ to the third $r$-process peak $(A \sim 195)$. There is also excellent agreement with observed abundances in metal-poor stars [71-73]. We find an overproduction at $A=132$ as observed in $[32,33]$. Below the second $r$-process peak, we recover the trends of the observed solar abundance pattern, but overall underproduce these nuclei, which is consistent with the absence of a significant high- $Y_{e}$ tail $Y_{e}>0.25$ (Fig. 4); however, a preliminary reanalysis including effects of neutrino absorption as in [69] indicates that the entire range of $r$-process nuclides can be obtained.

Conclusion.-We have shown that neutrino-cooled accretion disks in 3D GRMHD quickly self-regulate themselves into a state of moderate electron degeneracy (low $Y_{e}$ ) where heating from MRI-driven turbulence is balanced by neutrino cooling. The outflows launched selfconsistently as a result of this state tend to unbind more mass with a lower average $Y_{e}$ than previous axisymmetric Newtonian simulations employing an $\alpha$ viscosity. The nucleosynthesis yields show that these outflows are sufficiently neutron-rich to trigger a strong $r$ process and are well able to reproduce observed solar abundances and observed $r$-process abundances in metal poor stars from the second to the third $r$-process peak. Significant contributions to abundances below the second $r$-process peak can also come from BNS mergers leading to an accretion disk around a metastable hot neutron star, which, due to its strong neutrino emission, may raise $Y_{e}$ in part of the outflow material $[32,33,74]$.

We thank A. Beloborodov, R. Fernández, R. Haas, W. Kastaun, J. Lippuner, P. Moesta, C. Ott, and D. Radice for valuable discussions throughout the course of this work. Resources supporting this work were provided by the National Aeronautics and Space Administration (NASA) High-End Computing (HEC) Program through the NASA Advanced Supercomputing (NAS) Division at Ames Research Center; the work presented here consumed a total allocation worth $\approx 5.7 \mathrm{MCPUh}$. Support for this work was provided by NASA through Einstein Postdoctoral Fellowship Grant No. PF6-170159 issued by the Chandra X-ray Observatory Center, which is operated by the Smithsonian Astrophysical Observatory for and on behalf of the National Aeronautics Space Administration under Contract No. NAS8-03060. B.D.M. and D. M. S. acknowledge support from NASA ATP Grant No. NNX16AB30G and NSF Grant No. AST-1410950.

Note added.- - Recently a BNS merger has been detected by the Advanced LIGO and Virgo Collaborations [75]. The properties of the infrared kilonova emission observed from this event (inferred total ejecta mass $\approx 0.05 M_{\odot}$ and mean velocity $v \approx 0.1 c$, e.g., [76]) are consistent with the lanthanide-rich matter predicted in this work from disk outflows from a torus of initial mass $\approx 0.1 M_{\odot}$. 
*NASA Einstein Fellow.

[1] E. M. Burbidge, G. R. Burbidge, W. A. Fowler, and F. Hoyle, Rev. Mod. Phys. 29, 547 (1957).

[2] A. G. W. Cameron, Publ. Astron. Soc. Pac. 69, 201 (1957).

[3] Y.-Z. Qian and G. J. Wasserburg, Phys. Rep. 442, 237 (2007).

[4] M. Arnould, S. Goriely, and K. Takahashi, Phys. Rep. 450, 97 (2007).

[5] F.-K. Thielemann, A. Arcones, R. Käppeli, M. Liebendörfer, T. Rauscher, C. Winteler, C. Fröhlich, I. Dillmann, T. Fischer, G. Martinez-Pinedo, K. Langanke, K. Farouqi, K.-L. Kratz, I. Panov, and I. K. Korneev, Prog. Part. Nucl. Phys. 66, 346 (2011).

[6] E. Symbalisty and D. N. Schramm, Astrophys. Lett. 22, 143 (1982).

[7] J. M. Lattimer and D. N. Schramm, Astrophys. J. Lett. 192, L145 (1974).

[8] S. Rosswog, Int. J. Mod. Phys. D 24, 1530012 (2015).

[9] R. Fernández and B. D. Metzger, Annu. Rev. Nucl. Part. Sci. 66, 23 (2016).

[10] S. Rosswog, Astrophys. J. 634, 1202 (2005).

[11] R. Oechslin, H.-T. Janka, and A. Marek, Astron. Astrophys. 467, 395 (2007).

[12] A. Bauswein, S. Goriely, and H.-T. Janka, Astrophys. J. 773, 78 (2013).

[13] K. Hotokezaka, K. Kiuchi, K. Kyutoku, H. Okawa, Y.-i. Sekiguchi, M. Shibata, and K. Taniguchi, Phys. Rev. D 87, 024001 (2013).

[14] K. Kyutoku, K. Ioka, H. Okawa, M. Shibata, and K. Taniguchi, Phys. Rev. D 92, 044028 (2015).

[15] W. Kastaun and F. Galeazzi, Phys. Rev. D 91, 064027 (2015).

[16] D. Radice, F. Galeazzi, J. Lippuner, L. F. Roberts, C. D. Ott, and L. Rezzolla, Mon. Not. R. Astron. Soc. 460, 3255 (2016).

[17] M. Ruffert, H.-T. Janka, K. Takahashi, and G. Schaefer, Astron. Astrophys. 319, 122 (1997).

[18] M. Shibata and K. Taniguchi, Phys. Rev. D 73, 064027 (2006).

[19] R. Popham, S. E. Woosley, and C. Fryer, Astrophys. J. 518, 356 (1999).

[20] R. Surman, G. C. McLaughlin, M. Ruffert, H.-T. Janka, and W. R. Hix, Astrophys. J. 679, L117 (2008).

[21] B. D. Metzger, E. Quataert, and T. A. Thompson, Mon. Not. R. Astron. Soc. 385, 1455 (2008).

[22] L. Dessart, C. D. Ott, A. Burrows, S. Rosswog, and E. Livne, Astrophys. J. 690, 1681 (2009).

[23] A. Perego, S. Rosswog, R. M. Cabezón, O. Korobkin, R. Käppeli, A. Arcones, and M. Liebendörfer, Mon. Not. R. Astron. Soc. 443, 3134 (2014).

[24] S. Richers, D. Kasen, E. O’Connor, R. Fernández, and C. D. Ott, Astrophys. J. 813, 38 (2015).

[25] D. Martin, A. Perego, A. Arcones, F.-K. Thielemann, O. Korobkin, and S. Rosswog, Astrophys. J. 813, 2 (2015).

[26] B. D. Metzger, A. L. Piro, and E. Quataert, Mon. Not. R. Astron. Soc. 396, 304 (2009).

[27] W. H. Lee, E. Ramirez-Ruiz, and D. López-Cámara, Astrophys. J. 699, L93 (2009).

[28] A. M. Beloborodov, AIP Conf. Proc. 1054, 51 (2008).
[29] R. Fernández and B. D. Metzger, Mon. Not. R. Astron. Soc. 435, 502 (2013).

[30] R. Fernández, D. Kasen, B. D. Metzger, and E. Quataert, Mon. Not. R. Astron. Soc. 446, 750 (2015).

[31] O. Just, A. Bauswein, R. A. Pulpillo, S. Goriely, and H.-T. Janka, Mon. Not. R. Astron. Soc. 448, 541 (2015).

[32] M.-R. Wu, R. Fernández, G. Martínez-Pinedo, and B. D. Metzger, Mon. Not. R. Astron. Soc. 463, 2323 (2016).

[33] J. Lippuner, R. Fernández, L. F. Roberts, F. Foucart, D. Kasen, B. D. Metzger, and C. D. Ott, Mon. Not. R. Astron. Soc. 472, 904 (2017).

[34] S. A. Balbus and J. F. Hawley, Rev. Mod. Phys. 70, 1 (1998).

[35] M. Shibata, Y.-I. Sekiguchi, and R. Takahashi, Prog. Theor. Phys. 118, 257 (2007).

[36] http://einsteintoolkit.org.

[37] F. Löffler, J. Faber, E. Bentivegna, T. Bode, P. Diener, R. Haas, I. Hinder, B. C. Mundim, C. D. Ott, E. Schnetter, G. Allen, M. Campanelli, and P. Laguna, Classical Quantum Gravity 29, 115001 (2012).

[38] P. Mösta, B. C. Mundim, J. A. Faber, R. Haas, S. C. Noble, T. Bode, F. Löffler, C. D. Ott, C. Reisswig, and E. Schnetter, Classical Quantum Gravity 31, 015005 (2014).

[39] P. Colella and P. R. Woodward, J. Comput. Phys. 54, 174 (1984).

[40] A. Harten, J. Comput. Phys. 49, 357 (1983).

[41] B. Einfeldt, SIAM J. Numer. Anal. 25, 294 (1988).

[42] G. Tóth, J. Comput. Phys. 161, 605 (2000).

[43] P. Cerdá-Durán, J. A. Font, L. Antón, and E. Müller, Astron. Astrophys. 492, 937 (2008).

[44] F. X. Timmes and D. Arnett, Astrophys. J. Suppl. Ser. 125, 277 (1999).

[45] F. X. Timmes and F. D. Swesty, Astrophys. J. Suppl. Ser. 126, 501 (2000).

[46] K. A. van Riper and J. M. Lattimer, Astrophys. J. 249, 270 (1981).

[47] M. Ruffert, H.-T. Janka, and G. Schaefer, Astron. Astrophys. 311, 532 (1996).

[48] S. Rosswog and M. Liebendörfer, Mon. Not. R. Astron. Soc. 342, 673 (2003).

[49] Y. Sekiguchi, K. Kiuchi, K. Kyutoku, and M. Shibata, Phys. Rev. Lett. 107, 051102 (2011).

[50] C. D. Ott, E. Abdikamalov, P. Mösta, R. Haas, S. Drasco, E. P. O'Connor, C. Reisswig, C. A. Meakin, and E. Schnetter, Astrophys. J. 768, 115 (2013).

[51] A. Perego, R. M. Cabezón, and R. Käppeli, Astrophys. J. Suppl. Ser. 223, 22 (2016).

[52] F. Galeazzi, W. Kastaun, L. Rezzolla, and J. A. Font, Phys. Rev. D 88, 064009 (2013).

[53] D. Neilsen, S. L. Liebling, M. Anderson, L. Lehner, E. O'Connor, and C. Palenzuela, Phys. Rev. D 89, 104029 (2014).

[54] N. Stergioulas, J. Phys. Conf. Ser. 283, 012036 (2011).

[55] J.L. Friedman and N. Stergioulas, Rotating Relativistic Stars (Cambridge University Press, Cambridge, England, 2013).

[56] K. Kiuchi, Y. Sekiguchi, M. Shibata, and K. Taniguchi, Phys. Rev. D 80, 064037 (2009).

[57] L. Rezzolla, L. Baiotti, B. Giacomazzo, D. Link, and J. A. Font, Classical Quantum Gravity 27, 114105 (2010). 
[58] S. Bernuzzi, T. Dietrich, W. Tichy, and B. Brügmann, Phys. Rev. D 89, 104021 (2014).

[59] W. Kastaun, F. Galeazzi, D. Alic, L. Rezzolla, and J. A. Font, Phys. Rev. D 88, 021501 (2013).

[60] Y. Sekiguchi, K. Kiuchi, K. Kyutoku, M. Shibata, and K. Taniguchi, Phys. Rev. D 93, 124046 (2016).

[61] F. Foucart, Phys. Rev. D 86, 124007 (2012).

[62] K. Hotokezaka, K. Kyutoku, H. Okawa, M. Shibata, and K. Kiuchi, Phys. Rev. D 83, 124008 (2011).

[63] F. Foucart, D. Desai, W. Brege, M. D. Duez, D. Kasen, D. A. Hemberger, L. E. Kidder, H. P. Pfeiffer, and M. A. Scheel, Classical Quantum Gravity 34, 044002 (2017).

[64] K. Kiuchi, Y. Sekiguchi, K. Kyutoku, M. Shibata, K. Taniguchi, and T. Wada, Phys. Rev. D 92, 064034 (2015).

[65] R. Ciolfi, W. Kastaun, B. Giacomazzo, A. Endrizzi, D. M. Siegel, and R. Perna, Phys. Rev. D 95, 063016 (2017).

[66] A. M. Beloborodov, Astrophys. J. 588, 931 (2003).

[67] W. Chen and A. M. Beloborodov, Astrophys. J. 657, 383 (2007).

[68] J. Lippuner and L. F. Roberts, Astrophys. J. 815, 82 (2015).
[69] L. F. Roberts, J. Lippuner, M. D. Duez, J. A. Faber, F. Foucart, J. C. Lombardi, Jr., S. Ning, C. D. Ott, and M. Ponce, Mon. Not. R. Astron. Soc. 464, 3907 (2017).

[70] M. Arnould, S. Goriely, and K. Takahashi, Phys. Rep. 450, 97 (2007).

[71] C. Sneden, J. J. Cowan, J. E. Lawler, I. I. Ivans, S. Burles, T. C. Beers, F. Primas, V. Hill, J. W. Truran, G. M. Fuller, B. Pfeiffer, and K. Kratz, Astrophys. J. 591, 936 (2003).

[72] I. U. Roederer and J.E. Lawler, Astrophys. J. 750, 76 (2012).

[73] I. U. Roederer, J. E. Lawler, J. S. Sobeck, T. C. Beers, J. J. Cowan, A. Frebel, I. I. Ivans, H. Schatz, C. Sneden, and I. B. Thompson, Astrophys. J. Suppl. Ser. 203, 27 (2012).

[74] B. D. Metzger and R. Fernández, Mon. Not. R. Astron. Soc. 441, 3444 (2014).

[75] B. P. Abbott et al. (LIGO Scientific Collaboration and Virgo Collaboration), Phys. Rev. Lett. 119, 161101 (2017).

[76] P. S. Cowperthwaite et al., Astrophys. J. Lett. 848, L17 (2017). 